\title{
APPLICATION CLINIQUE DE LA TECHNIQUE DES PRODUITS DE DISTORSION ACOUSTIQUES AUDITIFS
}

\author{
J.C. BERA ${ }^{*, * *}$ et M. SUNYACH ${ }^{* *}$ \\ *Département de Physiologie Sensorielle: Audition et Voix, URA CNRS 1447, Hôpital Edouard \\ Herriot, France \\ ** Laboratoire de Mécanique des Fluides et d'Acoustique, CNRS URA-263, Ecole Centrale de Lyon, \\ BP. 163, F-69131 Ecully cedex, France
}

\begin{abstract}
To assess clinical utility of the distorsion product emission, 69 subjects were examined. Experiments showed in general a significant relation between the presence of distorsion products and the hearing losses. However the correlation coefficients did not exhibit higher values than $70 \%$. Therefore simple conclusions about the hearing threshold of an individual ear can't be drawn from a single distorsion product measurement.
\end{abstract}

\section{1 - INTRODUCTION :}

Les recherches effectuées récemment semblent mettre en évidence le caractère révéléteur des otoémissions acoustiques provoquées (OEAP) vis-à-vis du bon fonctionnement des mécanismes actifs cochléaires.

Parmi les OEAP, les produits de distorsion acoustiques semblent pouvoir jouer un rôle spécifique. D'une part il s'agit d'une méthode fréquentielle qui correspond à l'approche classique de l'audiogramme tonal, pour laquelle se trouve accumulé un grand nombre de données diagnostiques, d'autre part ses paramètres peuvent très facilement spécifiés et on peut mettre en rapport les résultats obtenus avec les données de la tonotopie cochléaire.

Nous présentons ici les résultats de nos propres expériences et de nos efforts en vue de simplifier la technique de mesure.

\section{2 - RELATION ENTRE LE PDA ET LES PERTES AUDITIVES :}

Les travaux récents sur les produits de distorsion [1], [2] permettent d'envisager l'hypothèse d'une application clinique en exploration fonctionnelle des PDA à la fréquence 2F1-F2. Rappelons que F1 et F2 désignent deux fréquences stimulantes dont le rapport est voisin de 1,22, comme le préconisent HARRIS et al. [3].

Dans nos propres travaux, nous avons utilisé une procédure désormais classique : les sujets à tester sont placés dans une chambre insonorisée, le manipulateur et les appareils (Analyseur HP 3561 A, synthétiseur bicanal HP 3326A) se trouvant à l'extérieur de la pièce. On a considéré une population de 136 oreilles et examiné la présence ou l'absence de PDA aux fréquences (F1/F2)/2 égales à 708, 1000, $1416,2000,2832,4000,5664 \mathrm{~Hz}$.

Nos principaux résultats (qui sont détaillés par ailleurs dans BERA, [4]) sont les suivants :

19) Le groupe sans PDA (11 \%) présente les pertes auditives les plus importantes (Fig. 1.a) avec une dispersion assez forte.

29) Le groupe avec PDA à toutes les fréquences (25\%) correspond à une population plus jeune, avec une audition normale ou presque normale (Fig. 1.b). 
39) L'audiogramme moyen suggère l'existence d'une corrélation marquée entre les pertes auditives et l'absence de PDA (Fig. 2).

D'autres informations peuvent être apportées par l'étude des seuils : on désigne par seuil de détection le niveau des pressions des primaires $\mathrm{F} 1$ et $\mathrm{F} 2$, à partir duquel le PDA dépasse le bruit de fond de $3 \mathrm{~dB}$. On désigne par seuil d'intensité le niveau atteint par le PDA à un niveau primaire donné $(70 \mathrm{~dB})$. Les résultats présentés sur les figures 3.a et 3.b indiquent les valeurs extrêmes du nuage de points représentant la population testée, les quarticles et la médiane. Les valeurs maximum des corrélations correspondantes vont de $67 \%$ pour le seuil de détection des PDA et $50 \%$ pour celui de l'intensité.

Une étude similaire effectuée en basse fréquence, en-dessous de $700 \mathrm{~Hz}$, montre des corrélations moins fortes. Ces basses fréquences peuvent donc impliquer des mécanismes particuliers [5].

La forme triangulaire des diagrammes des figures 3.a et 3.b montre une relation unilatérale entre les PDA et les pertes auditives : ces dernières constituent donc un facteur limitatif des PDA. Il est donc clair que si la présence de ces derniers renseigne sur le fonctionnement de l'amplificateur dynamique cochléaire, d'autres mécanismes complexes entrent en jeu : la prédiction de l'audiogramme tonal à partir des PDA ne semble pas réalisable dans tous les cas : la technique peut apporter des renseignements précis et fiables mais son interprétation n'est pas possible sans autres informations.

\section{3 - REALISATION D'UN APPAREILLAGE SPECIFIQUE POUR LA MESURE DES PDA}

Nous avons rassemblé dans un même module le système d'émission des deux fréquences $\mathrm{F} 1$ et $\mathrm{F} 2$ et une voie d'acquisition et d'analyse FFT. Ce module est conçu pour être piloté par un micro-ordinateur de type P.C. Il est composé de deux microprocesseurs de traitement du signal ADSP 2101 et 2105 , l'un prenant en charge la partie émission (synthétiseur) l'autre la partie réception et FFT. Ces deux microprocesseurs communiquent entre eux par une liaison série ce qui permet d'ajuster les niveaux d'émission. Deux possibilités de moyennage sont prévues : la première est un moyennage classique qui accumule l'énergie dans les bandes d'analyse, le second est un moyennage synchrone qui moyenne la signature de pression et permet donc d'accentuer les fréquences qui sont en relation de phase déterministe avec l'émission. La dynamique d'ensemble (entrée/sortie) permet de mesurer l'émergence d'une fréquence pure jusqu'à $100 \mathrm{~dB}$.

On s'est essentiellement intéressé aux possibilités de mesure des PDA dans un environnement autre qu'une chambre acoustique isolée. La matériel et l'opérateur peuvent alors être en présence du sujet à tester. Des résultats typiques sont représentés sur la figure 4.

Dans une pièce calme (niveau acoustique de $36 \mathrm{dBA}$ contre $26 \mathrm{dBA}$ dans un local isolé acoustiquement), le moyennage classique fait par exemple apparaître un PDA de $8 \mathrm{~dB}$ pour un niveau de primaire moyen de $68 \mathrm{~dB}$ à la fréquence de $2,6 \mathrm{kHz}$ (Fig. 4.a).

L'utilisation d'une acquisition synchronisée augmente alors notablement l'émergence du signal des PDA. Elle est ainsi de $10 \mathrm{~dB}$ pour 16 moyennes (Fig. 4.b) et de $15 \mathrm{~dB}$ pour 32 moyennes (Fig. 4.c). La limite de l'utilisation de ce procédé peut être observée sur les figures 4.d et 4.e. Pour un niveau ambiant de $46 \mathrm{dBA}$ (bruit de ventilation des appareils de mesure classiques). Un moyennage synchrone (16 acquisitions) permet à peine de détecter le PDA (Fig. 4.e). Ces résultats peuvent naturellement être obtenus dans toute la gamme de fréquences. Il semble donc que ce type d'appareillage peut permettre de simplifier et de faire progresser la technique et l'interprétation des PDA. 


\section{BIBLIOGRAPHIE}

[1] Lonsbury-Martin \& al., Annals of otology, thinology et laryngology, Suppl. 147, 99, 5 (1990).

[2] Probst R., Second Symposium on clinical applications of otoacoustic emissions, Lyon, (1989).

[3] Harris F.P., et al., J. Acoust. Soc. Am., 85, 1 (1989).

[4] Béra J.C., DEA d'Acoustique, ECL (1990).

[5] Bonfils P. \& Al., Acta Otolaryngol. Head Neck Surg., 115 (1989).
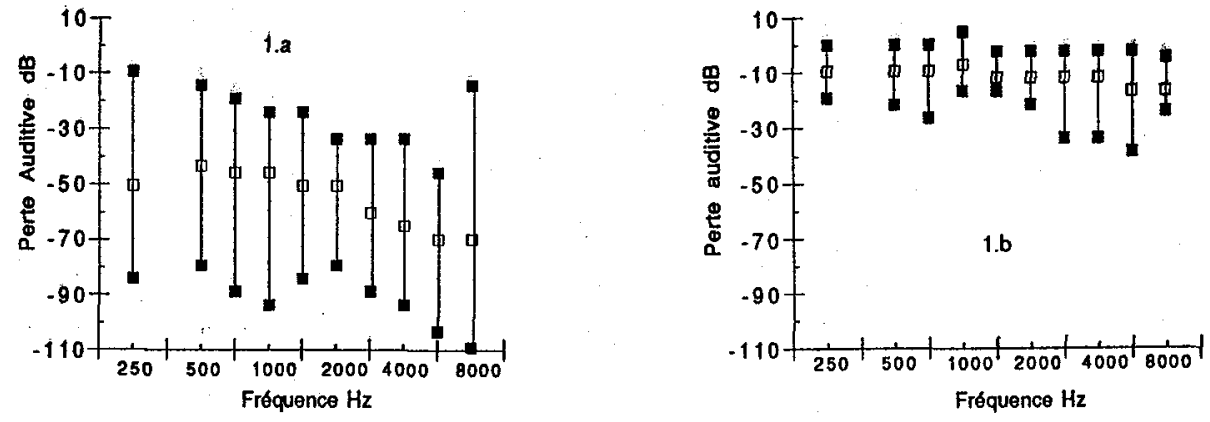

Fig. 1.a : Audiogramme du groupe sans P.D.A.

Les points $\square$ représentent les valeurs extrêmes,

Fig. 1.b : Audiogramme du groupe les points $\square$ la moyenne.

présentant des P.D.A.

à toutes les fréquences.

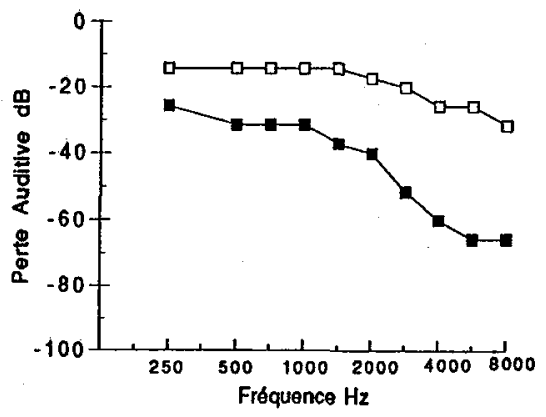

Fig.2 : Audiogramme moyen du groupe sans PDA et du groupe avec PDA $\square$, à la frequence de $2000 \mathrm{~Hz}$. 

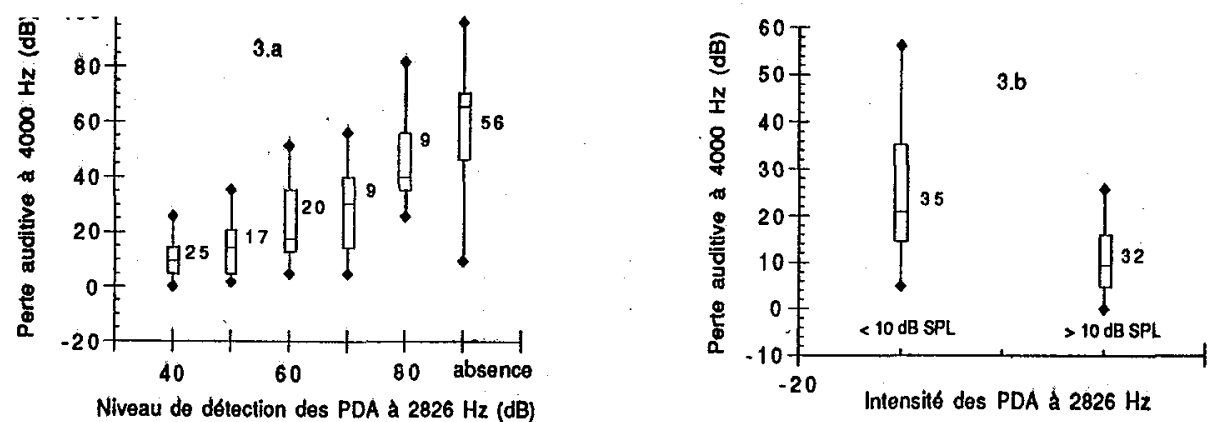

Niveau de détection des PDA à $2826 \mathrm{~Hz}$ (dB)

Fig. 3 : Répartition statistique des pertes auditives à $4000 \mathrm{~Hz}$

a) en fonction du niveau des primaires

b) en fonction des seuils d'intensité.

Les chiffres indiquent le nombre d'oreilles; les boites les quartiles, le trait la moyenne, le point - les valeurs extrêmes.
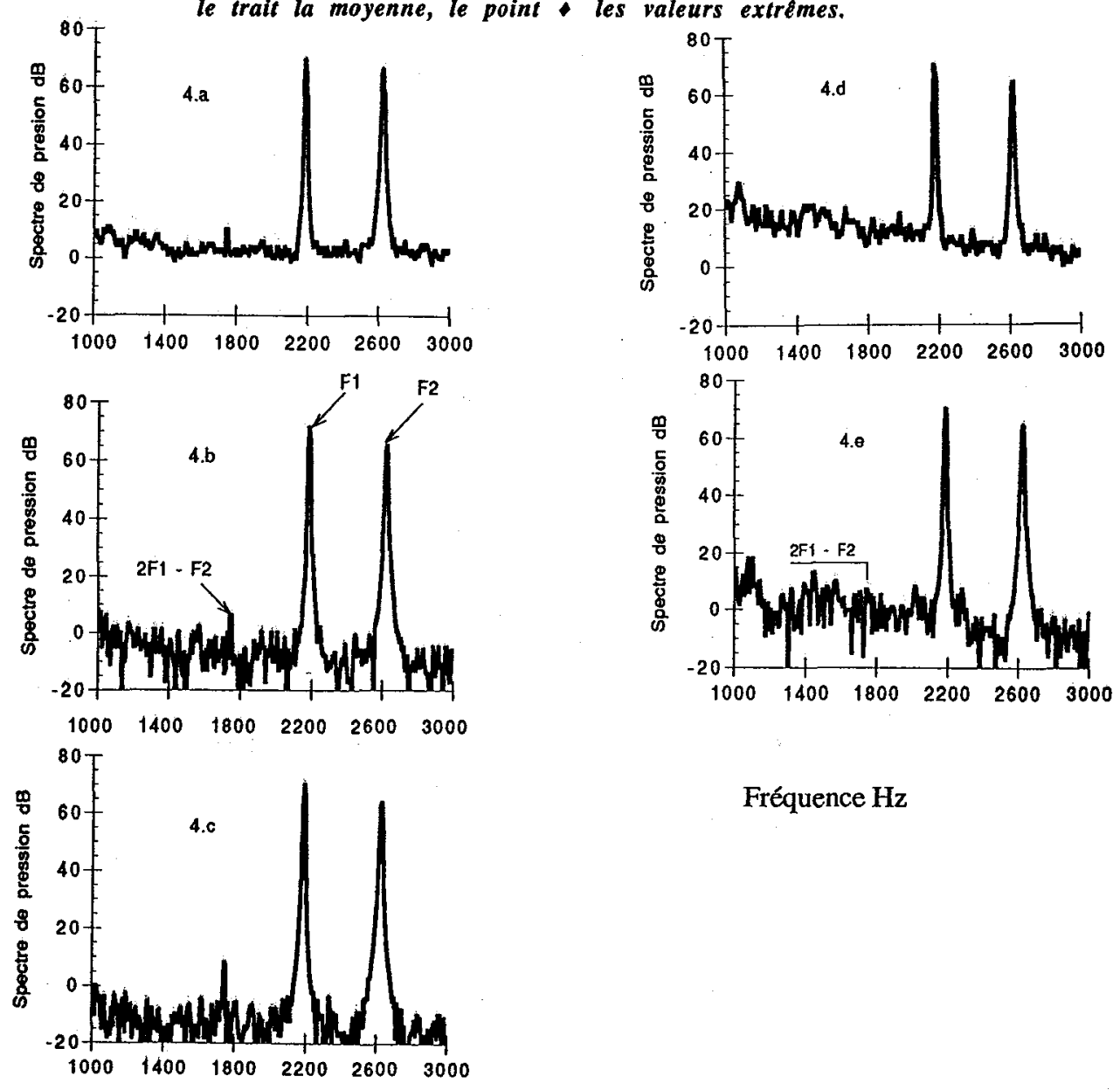

Fréquence $\mathrm{Hz}$

Fig. 4 : Mesure de produits de distorsion acoustiques

a) moyennage classique, niveau de bruit ambiant: 26 dBA (8 moyennes)

b) moyennage synchronisé niveau de bruit ambiant: 26 dBA (16 moyennes)

c) moyennage synchronisé niveau de bruit ambiant: 26 dBA (32 moyennes)

d) moyennage classique, niveau de bruit ambiant de $46 \mathrm{dBA}$ ( 8 moyennes)

e) moyennage synchronise niveau de bruit ambiant de 46 dBA (16 moyennes) 